\title{
Administração pública: o pacto pela saúde como uma nova estratégia de racionalização das ações e serviços em saúde no Brasil*
}

\author{
Cristina Berger Fadel** \\ Luciana Schneider*** \\ Suzely Adas Saliba Moimaz**** \\ Nemre Adas Saliba*****
}

SumÁrio: 1. Introdução; 2 . As principais tentativas para a operacionalização do SUS: breve histórico; 3 . Pactuando na saúde: uma oportunidade de avanço estratégico; 4. Considerações finais.

Summary: 1. Introduction; 2. Key attempls to operationalize the Unified Health System: brief history; 3. Making a health pact: an opportunity for strategic development; 4. Final remarks.

Palavras-chave: política de saúde; Sistema Único de Saúde; organização \& administração.

KEY wORDs: health policy; Unified Health System; organization \& management.

\footnotetext{
* Artigo recebido em jul. 2007 e aceito em mar. 2008.

$\because *$ Professora assistente do Departamento de Odontologia da Universidade Estadual de Ponta Grossa, doutoranda em odontologia social e preventiva pelo Departamento de Odontologia Infantil e Social da Faculdade de Odontologia de Araçatuba da Universidade Estadual Júlio de Mesquita Filho. Endereço: Rua Paula Xavier, 909, ap. 124 - Centro - CEP 84010-270, Ponta Grossa, PR, Brasil. E-mail: cfadel@itelefonica.com.br.

$* * *$ Mestranda em odontologia social e preventiva pelo Departamento de Odontologia Infantil e Social da Faculdade de Odontologia de Araçatuba da Universidade Estadual Júlio de Mesquita Filho. E-mail: esodontofederal@yahoo.com.br.

$* * * *$ Professora doutora adjunta do Departamento de Odontologia Infantil e Social da Faculdade de Odontologia de Araçatuba da Universidade Estadual Júlio de Mesquita Filho. Endereço: Unesp — Rua José Bonifácio, 1193 - Vila Mendonça - CEP 16015-050, Araçatuba, SP, Brasil. E-mail: ssaliba@foa.unesp.br.

$\because * * * *$ Professora doutora titular do Departamento de Odontologia Infantil e Social da Faculdade de Odontologia de Araçatuba da Universidade Estadual Júlio de Mesquita Filho. Endereço: Unesp — Rua José Bonifácio 1193 — Vila Mendonça — CEP 16015-050, Araçatuba, SP, Brasil. E-mail: nemre@foa.unesp.br.
} 
O pacto pela saúde surge no cenário brasileiro após inúmeras tentativas de operacionalização do Sistema Único de Saúde (SUS), em busca da consolidação da equidade social. Este artigo divulga o pacto, visando subsidiar o processo de administração pública no Brasil, por meio de análise documental. Essa nova política, ainda em fase inicial de implementação, constitui uma realidade única e altamente viável à otimização das práticas nacionais em saúde pública, estando o seu cumprimento diretamente relacionado à transposição de entraves políticos e operacionais inerentes a cada nível de gestão.

Public administration: the health pact as a new strategy to rationalize healthcare actions and services in Brazil

The health pact arises in the Brazilian scene after a number of attempts for the improvement of the Unified Health System (SUS), so as to consolidate social equity. In this perspective, this paper discusses the pact using documental analysis, in order to help the public administration process in Brazil. This new policy, still in the initial phase of implementation, is a unique reality and highly feasible for the improvement of national practices in public health. Its performance is intimately related to overcoming the political barriers inherent to each rank of the administration.

\section{Introdução}

A importância do reconhecimento de estratégias consolidadoras do Sistema Único de Saúde (SUS) vem crescendo paulatinamente no Brasil, tanto sob a perspectiva constitucional, relativa à conquista de direitos individuais e coletivos; quanto ao campo das ações, enfatizando-se a valorização das dimensões que envolvem a participação popular nos processos decisórios em saúde.

Inúmeras e constantes são as tentativas de ampliação de mecanismos que levem à otimização dos serviços públicos de saúde e ao acesso dos usuários a todos os níveis de atenção do sistema, entretanto, a questão assume uma forte complexidade ao considerarem-se as enormes desigualdades geográficas, culturais e socioeconômicas que há tempos permeiam o país.

A Lei no 8.080, de 19 de setembro de 1990 (Brasil, 1990a), expressou os avanços incorporados na Constituição de 1988 (Brasil, 1988), que admitiam ser a saúde, em sua perspectiva ampliada, um direito da população, e ainda, a universalidade e a equidade do acesso aos serviços de saúde, a integralidade das ações praticadas, o intenso controle social e a descentralização político-administrativa princípios norteadores do sistema de saúde vigente no Brasil (Brasil, 1990b). Sob essa perspectiva, sem dúvida, a descentralização dos serviços de saúde, entendida como parte do processo de reforma democrática do Estado, evidenciou-se como uma prática legítima e, em grande parte, viabilizadora das políticas públicas nacionais (Roncalli, 2003), uma vez que a quebra da hegemonia estatal abriu um importante caminho para os primeiros 
passos de uma gestão participativa, com urgentes e necessários embates políticos e sanitários entre os diversos atores sociais envolvidos.

Nesse contexto, permeado por tentativas que, embora tenham cumprido seu papel no processo histórico de evolução do SUS, não foram resolutivas, este artigo divulga o pacto pela saúde, visando subsidiar o processo de gestão em saúde no Brasil, por meio da análise de documentos oficiais que regulamentam as políticas públicas no país.

\section{As principais tentativas para a operacionalização do SUS: breve histórico}

Apesar da conquista de um embasamento jurídico sólido para a legitimação do SUS, por meio, principalmente, de leis orgânicas, suscitou-se ainda a necessidade de regulamentação prática do sistema, com algo que claramente dispusesse sobre as ações cotidianas em saúde.

Primeiramente, incidiram sobre o SUS normas reguladoras, denominadas normas operacionais básicas (NOB), criadas para operar as transformações previstas na Constituição e na Lei Orgânica da Saúde (Carvalho, 2001). Estas, cada qual com contribuições particulares para os avanços na saúde, foram sequencialmente editadas por órgãos de competência. Assim, o sistema de saúde brasileiro perpassou por: NOB Inamps/91 (Brasil, 1991), SUS/93 (Brasil, 1993) e SUS/96 (Brasil, 1996).

Em cada NOB, novas formas de relação entre os gestores foram introduzidas e, com elas, novos formatos de alocação de recursos no setor. A descentralização da política da saúde foi acontecendo gradativamente, assim como a do seu financiamento (Mansur, 2001). Portanto, a cada novo passo do SUS, surgia uma forma distinta de participação dos estados e municípios e uma nova forma de transferência de receita da União para eles.

A primeira NOB (Brasil, 1991) nasceu dentro do extinto Instituto Nacional de Assistência Médica da Previdência Social (Inamps) e representou basicamente os interesses dessa autarquia, ao dar continuidade ao modelo já instituído de repasses financeiros estaduais e municipais por produção de serviços e à prática de acompanhamento, controle e avaliação de todas as ações.

A NOB SUS/93 (Brasil, 1993), publicada após a realização da IX Conferência Nacional de Saúde, que enfatizou a necessidade da municipalização, trouxe importantes mudanças no cenário estabelecido até então (Romano, 1998). A instituição de formas de gestão coparticipativas (incipiente, parcial e semiplena) foi estimulada, de acordo com o nível organizacional e de comprometimento com o SUS de cada município (Mendes, 1998), representando um nível diferente e progressivo de transferência de responsabilidades e au- 
tonomia de gestão (Mansur, 2001). A adesão dos municípios a esse processo de descentralização deu-se de maneira bastante intensa, fato que resultou no enfraquecimento dos estados, vistos agora como coadjuvantes e não mais protagonistas na gestão dos serviços de saúde pública brasileiros.

Com o processo de municipalização crescendo substancialmente, especialmente em nível da atenção básica (Marques e Mendes, 2002), a NOB SUS/96 (Brasil, 1996) vem ao encontro do equilíbrio entre os atores sociais envolvidos com a gestão do SUS, induz à estratégia de descentralização (Viana et al., 2002) e redefine as responsabilidades dos estados e da União. O poder público estadual assume a mediação das relações entre municípios e esfera federal, ou, provisoriamente, a gestão municipal, até que o município apresente autonomia para apropriar-se de um sistema adequado. Um dos pontos de maior destaque relacionou-se ao financiamento, com a criação de novos subtetos de recursos, como o piso da atenção básica — valor per capita para atenção básica universal a todos os municípios - e recursos específicos para financiar o Programa de Agentes Comunitários de Saúde (Pacs) e o Programa de Saúde da Família (PSF), e com a redução progressiva da remuneração por produção de serviços.

Apesar dos indiscutíveis avanços oriundos do processo de descentralização do sistema de saúde no Brasil, especialmente como condição necessária para melhorar o acesso aos serviços de saúde (Hortale, Pedroza e Rosa, 2000), algumas debilidades resultantes desse "desenvolver em saúde" foram apontadas. A fragmentação da integralidade das ações e o enfraquecimento gradativo dos estados, que ainda contavam com uma pobre participação no crescimento das ações, obtiveram destaque (Mansur, 2001; Mendes, 1998).

Inicialmente como uma medida de cunho muito mais político do que organizacional, é publicada no Brasil a primeira norma operacional de assistência à saúde - Noas SUS 01/01 (Brasil, 2001). Essa norma aponta para 0 reconhecimento da regionalização da atenção à saúde como uma macroestratégia para consolidação dos princípios da universalidade, equidade e integralidade; constituindo-se um passo a mais em direção à descentralização. Agora, os gestores municipais devem ser capazes de ofertar, além de serviços inerentes à atenção básica, também o acesso aos serviços de maior complexidade, na tentativa de organizar a demanda regionalmente. Vale lembrar que o financiamento para esses novos serviços é dado pela ampliação do valor fixo do $\mathrm{PAB}$, criando o conceito de atenção básica ampliada.

A insuficiência de mecanismos para a incorporação das novidades trazidas pela Noas SUS 01/01 (Brasil, 2001) e a necessidade de assegurar a manutenção das diretrizes organizativas anteriormente definidas culminam 
com a publicação da Noas SUS 01/02 (Brasil, 2002), que procura oferecer alternativas viáveis à superação dos entraves oriundos da dinâmica concreta de sua implementação, por meio, principalmente, da regulação da assistência, agora apresentada de forma mais completa.

As condições de gestão estabelecidas pela Noas 01/02 (Brasil, 2002) explicitam as responsabilidades do gestor municipal, os requisitos relativos às modalidades de gestão: gestão plena da atenção básica ampliada e gestão plena do sistema municipal e as prerrogativas municipais e estaduais, ao exercer o papel de fio condutor clássico capaz de viabilizar, por meio do financiamento, a política pública (Marques e Mendes, 2002). A habilitação por essa norma pressupõe que estados e municípios organizem suas estruturas de controle, regulação e avaliação visando à garantia de ações integrais e resolutivas, capazes de verdadeiramente impactar sobre os problemas de saúde da população. Sem dúvida, mostra-se como o movimento mais forte em direção ao processo de regionalização, com estratégia de hierarquização dos serviços de saúde e da busca de maior equidade, com a criação de mecanismos para fortalecimento da capacidade de gestão do Sistema Único de Saúde e com a atualização dos critérios de habilitação de estados e municípios.

Para a qualificação na Noas 01/02 (Brasil, 2002), os estados devem elaborar o Plano Diretor de Regionalização (PDR), que inclui o Plano Diretor de Investimento (PDI) e a Programação Pactuada e Integrada (PPI). O PDI deve estabelecer, de acordo com as ações pactuadas nos âmbitos regional e estadual na PPI, os recursos necessários à sua implementação e desenvolvimento, garantindo o financiamento integral da atenção básica e das necessidades no setor de vigilância à saúde (Brasil, 2002). No entanto, apesar dos esforços das três esferas de gestão na implementação da Noas 01/02 (Brasil, 2002), observa-se certo descompasso nesse processo, uma vez que nem todos os estados se encontram no mesmo momento de evolução, surgindo, assim, a necessidade de uma nova estratégia de operacionalização do SUS no Brasil.

\section{Pactuando na saúde: uma oportunidade de avanço estratégico}

Visando à superação desse desacordo evolutivo entre os estados, é editada a Portaria GM/MS no 399, de 22 de fevereiro de 2006 (Brasil, 2006a), que divulga o pacto pela saúde - consolidação do SUS e aprova as diretrizes operacionais inerentes a ele.

O pacto pela saúde (PS) surge no cenário brasileiro em um momento histórico da saúde pública no país. Após as inúmeras tentativas de transpo- 
sição dos entraves regionais e operacionais no setor saúde, por meio da luta pela descentralização, regionalização e hierarquização das ações e serviços, o Brasil aponta hoje como palco do apogeu desse processo em busca da equidade social na saúde. Como uma oportunidade, aos gestores federados no âmbito do SUS, de avanço estratégico nessa área, o PS visa à qualificação e implementação do processo de descentralização, organização e gestão do sistema de saúde do Brasil à luz da evolução e aprimoramento do processo de pactuação intergestores. Entre seus objetivos, destacam-se elencar, aperfeiçoar e definir responsabilidades sanitárias e de gestão por meio de três componentes: o pacto pela vida (PV), o pacto em defesa do SUS (Pdsus) e o pacto de gestão (PG), como mostra a figura 1 (Brasil, 2006a).

\section{Figura 1}

\section{Pacto da saúde: uma estratégia para viabilizar a equidade em saúde no Brasil.}

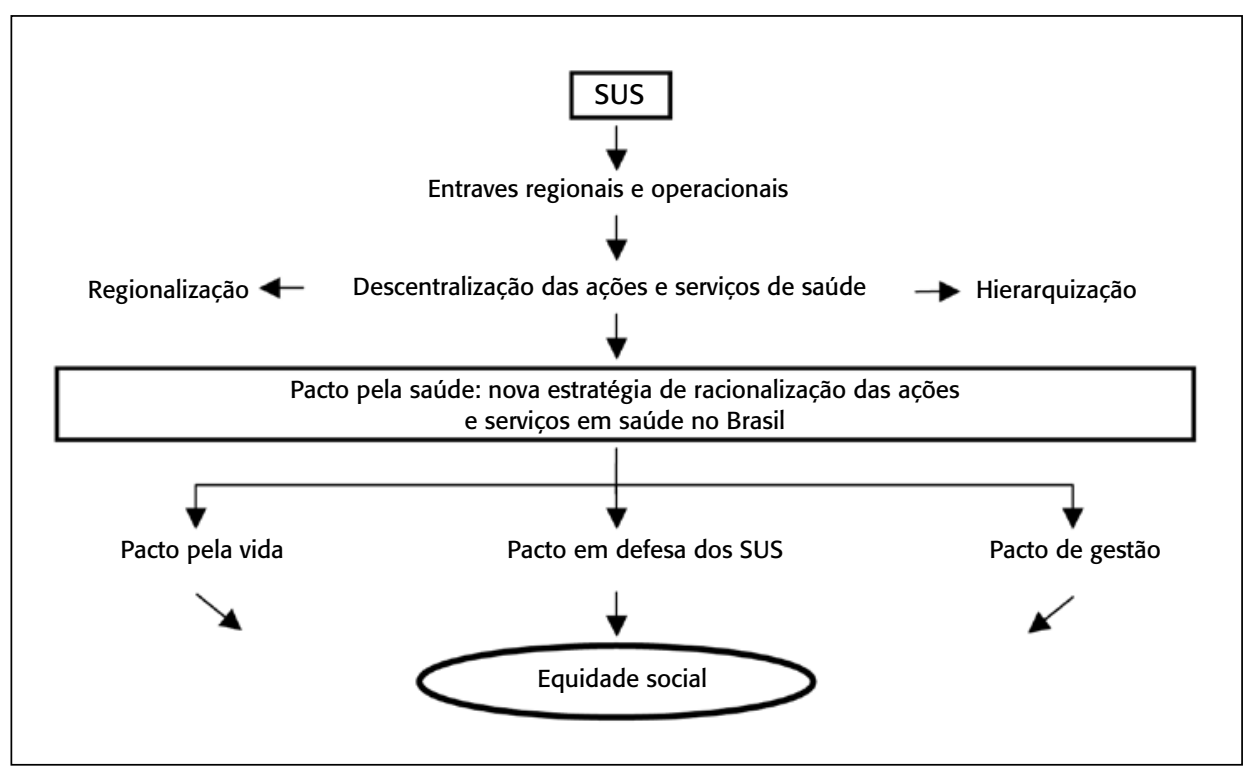

\section{O pacto pela vida}

O PV é "o compromisso entre os gestores do SUS em torno de prioridades, estabelecidas através de metas nacionais, estaduais ou municipais, que apresentam impacto sobre a situação de saúde da população brasileira" (Brasil, 2006a). As prioridades pactuadas apresentam-se divididas em seis grandes 
vertentes: a saúde do idoso, o controle de câncer de colo de útero e de mama, a redução da mortalidade infantil e materna, o fortalecimento de capacitação de respostas às doenças emergentes e endemias com ênfase na dengue, hanseníase, tuberculose, malária e influenza, a promoção da saúde e o fortalecimento da atenção básica (Brasil, 2006a).

Desses grupos, destaca-se a promoção da saúde, cujo objetivo primeiro é o da elaboração e implementação de uma política ampla que contemple as diversas faces desse campo, em seus entraves e debilidades, e que ratifique a responsabilidade individual sobre a saúde, valendo-se de seu conceito ampliado (Brasil, 1990c, 1998). No âmbito político, visa à pactuação da política nacional de promoção da saúde, a partir da realidade de ações e serviços de saúde dos estados e municípios (Brasil, 2006a).

Ressalta-se também o fortalecimento da atenção básica por meio do apoio instituído aos diferentes modos de organização da atenção, ao priorizar o PSF, a sua consolidação em grandes centros e a ampliação dele em municípios de pequeno e médio portes, respeitando suas diferenças regionais (Brasil, 2006b). Quanto ao financiamento e à infraestrutura, garante o funcionamento das unidades básicas de saúde (UBSs) a partir de recursos provenientes das três esferas de gestão do SUS (Brasil, 2000, 2006a, 2006b, 2006c, 2006d, 2006e), devendo ser monitorado e avaliado com vistas à qualificação da gestão descentralizada (Brasil, 2006a, 2006f). Em relação aos recursos humanos necessários, aprimora a inserção dos profissionais nas redes locais de saúde, por meio de vínculos de trabalho que favoreçam a sua fixação, e qualifica-os por meio de estratégias de educação permanente e da oferta de cursos de caráter continuado, como especializações e residências multiprofissionais em saúde da família (Brasil, 2006b).

\section{O pacto em defesa do SUS}

O Pdsus expressa "o compromisso entre os gestores do SUS com o desenvolvimento e a articulação de ações que visem qualificar e assegurar o SUS como política pública" (Brasil, 2006a). Assim, por meio de iniciativas como a repolitização da saúde, com a retomada das discussões sobre os desafios atuais e futuros do SUS, as novas modalidades de gestão, a ampliação e o fortalecimento das relações com os movimentos sociais em prol da saúde e da cidadania, o controle social e ainda a transparência de seu processo de fiscalização (Brasil, 2006a), como instrumentos para uma construção coparticipativa do sistema (Brasil, 1988; 1990a, 1990c), esse pacto vem ao encontro das expectativas há 
tempo vivenciadas pelo setor, no que se refere ao acolhimento da inserção da população no processo decisório em saúde.

\section{Pacto de gestão: uma alternativa real para o processo de administração em saúde no Brasil}

O PG estabelece "as diretrizes para a gestão do sistema nos aspectos de descentralização, regionalização, financiamento, planejamento, programação pactuada e integrada, regulação, participação social e gestão do trabalho e da educação na saúde" (Brasil, 2006a). Assim, esse pacto concorre positivamente aos legítimos anseios gerados pelo extinto movimento sanitário, em busca da equidade social na saúde, uma vez que é uma alternativa real de avanços nas ações e serviços de saúde no Brasil.

Aprofundando-se um pouco mais no âmbito da gestão, um campo repleto de normativas a serem percorridas, torna-se importante salientar que a organização, a direção e a gestão do SUS devem ser exercidas concomitantemente pelos conselhos de saúde e pelo poder executivo, em cada esfera de governo (Brasil, 1990a, 1990c). Vale novamente ressaltar o caráter conjuntivo que a ele se relaciona, uma vez que a articulação entre os gestores nos diferentes níveis de atuação viabiliza-se por meio de dois colegiados de negociação: a Comissão Intergestores Tripartite (CIT) e a Comissão Intergestores Bipartite (CIB). A CIT é constituída de forma paritária, por representantes do Ministério da Saúde (MS) e do Conselho Nacional de Secretários de Saúde (Conass). Já a CIB, também paritariamente formada, integra-se por entes da Secretaria Estadual de Saúde (SES) e do Conselho Estadual de Secretários Municipais de Saúde (Coness). No espaço reservado às atribuições de cada esfera, compete ao MS a proposição de políticas, a participação no cofinanciamento, a cooperação técnica, a avaliação, a regulação, o controle e a fiscalização. À CIT, cabe a pactuação de diretrizes e normas a serem acordadas e deliberadas pela CIB, à qual é atribuída a disposição máxima sobre os termos operacionais (figura 2).

O princípio da regionalização, assegurado pela Constituição Federal (Brasil, 1988) e pela Lei no 8.080 (Brasil, 1990a), adquire a função de eixoestruturante do PG. Segundo ele, as unidades pactuadas são "recortes territoriais inseridos em um espaço geográfico contínuo, identificadas pelos gestores municipais e estaduais a partir de identidades culturais, econômicas e sociais, de redes de comunicação e infraestrutura de transportes compartilhados do território" (Brasil, 2006a), cuja composição deve acatar os seguintes critérios: contiguidade entre os municípios, respeito à identidade expressa no cotidiano 
social, econômico e cultural; existência de infraestrutura de transportes e de redes de comunicação; existência de fluxos assistenciais que devam ser alterados, se necessário, para a organização da rede de atenção à saúde (Brasil, 2006a).

Figura 2

Pacto de gestão: direção e articulação do SUS nas três esferas de governo

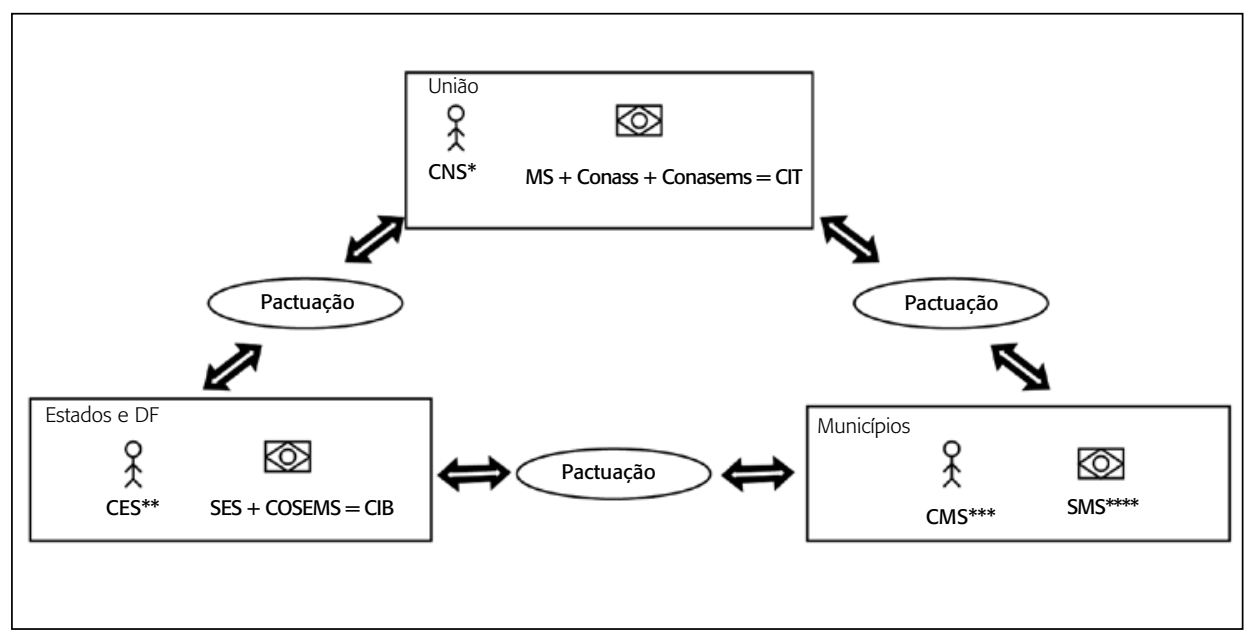

*CNS - Conselho Nacional de Saúde; **CES - Conselho Estadual de Saúde; ***CMS - Conselho Municipal de Saúde; ****SMS - Secretaria Municipal de Saúde.

O Dicionário Larousse, ao definir pacto como "um encontro de vontades com o objetivo de produzir efeitos de direito desejados pelas partes", encerra o mesmo conceito designado a esse termo pelas diretrizes operacionais do PG, que o concebe como "um processo que visa definir as programações de saúde em cada território e nortear a alocação dos recursos financeiros para a saúde a partir de critérios e parâmetros pactuados entre os gestores" (Brasil, 2006a). Nesse contexto, destacam-se, entre as diretrizes norteadoras do processo de pactuação, a flexibilidade no planejamento, respeitando as prioridades fixadas pelos planos de saúde em cada esfera de gestão; a submissão à ordem hierárquica entre as ações básicas e às de maior complexidade; a integração com a programação da vigilância em saúde; a garantia de acesso aos serviços de saúde, subsidiando o processo regulatório da assistência; a avaliação periódica ao desenvolvimento das ações (Brasil, 2006a). Vale ressaltar ainda que a PPI deve guardar íntima relação com o desenho da regionalização a qual está vinculada, assumindo e moldando-se às suas características. 
No que se relaciona ao financiamento, mantém-se a corresponsabilidade das três esferas de gestão e o repasse fundo a fundo como modalidade preferencial de transferência de recursos entre os gestores (Brasil, 2000, 2006a, 2006b, 2006c, 2006d, 2006e). Entretanto, o processo de pactuação permite uma maior maleabilidade na utilização dos recursos em saúde, uma vez que opera sobre bases concretas, previamente produzidas e combinadas pelas partes.

Nessa perspectiva, o PG surge na atual conjuntura brasileira como uma oportunidade real de melhoria no acesso e na qualidade dos serviços e de resolução dos problemas operacionais não equacionados com as NOB e as Noas, possibilitando assim a redução das iniquidades macrorregionais, estaduais e regionais, no que diz respeito às ações e serviços em saúde.

\section{Considerações finais}

Não resta dúvida que o pacto pela saúde constitui, atualmente, a mais nova estratégia para a racionalização das ações e serviços em saúde no Brasil, uma vez que debruça e norteia suas condutas sobre a equidade social.

Essa nova política, ainda em fase inicial de implementação, é uma realidade única e altamente viável à otimização das práticas nacionais em saúde pública, estando o seu cumprimento diretamente relacionado à transposição de entraves políticos e operacionais inerentes a cada nível de gestão.

Vale ressaltar que as prerrogativas e responsabilidades dos municípios e estados habilitados em gestão plena do sistema devem ser mantidas, ainda sob a regulamentação das normas vigentes - NOB SUS/96 (Brasil,1996) e Noas SUS 01/02 (Brasil, 2002), até que a unificação total do processo ocorra.

\section{Referências bibliográficas}

BRASIL. Constituição Federal, de 5 de outubro de 1988.

. Lei no 8.080 . Dispõe sobre as condições para a promoção, proteção e recuperação da saúde, a organização e o funcionamento dos serviços correspondentes e dá outras providências. Diário Oficial da União, 20 set. 1990a.

. Ministério da Saúde. Secretaria Nacional de Assistência à Saúde. $A B C$ do SUS: doutrinas e princípios. Brasília, 1990b. 
. Lei no 8.142. Dispõe sobre a participação da comunidade na gestão do Sistema Único de Saúde (SUS) e sobre as transferências intergovernamentais de recursos financeiros na área da saúde e dá outras providências. Diário Oficial da União, 31 dez. 1990c.

. Ministério da Saúde. Resolução no 273/91. Norma Operacional Básica do Instituto Nacional de Assistência Médica da Previdência Social: NOB-Inamps 01/91. Diário Oficial da União, 7 jan. 1991.

. Ministério da Saúde. Portaria no 545. Norma Operacional Básica do Sistema Único de Saúde: NOB-SUS 01/93. Diário Oficial da União, 24 maio 1993.

. Ministério da Saúde. Portaria no 2.203. Norma Operacional Básica do Sistema Único de Saúde: NOB-SUS 01/96. Gestão plena com responsabilidade pela saúde do cidadão. Diário Oficial da União, 6 nov. 1996.

. Ministério da Saúde. Emenda Constitucional no 29. Altera os arts. 34, 35, 156, 160, 167 e 198 da Constituição Federal e acrescenta artigo ao Ato das Disposições Constitucionais Transitórias, para assegurar os recursos mínimos para o financiamento das ações e serviços públicos da saúde. Diário Oficial da União, 14 set. 2000.

. Ministério da Saúde. Portaria no 95. Norma Operacional de Assistência à Saúde: NOAS-SUS 01/01. Diário Oficial da União, 26 jan. 2001.

. Ministério da Saúde. Portaria no 373. Norma Operacional de Assistência à Saúde: NOAS-SUS 01/02. Diário Oficial da União, 28 fev. 2002.

. Ministério da Saúde. Portaria no 399. Divulga o Pacto pela Saúde 2006 - Consolidação do SUS e aprova as Diretrizes Operacionais do Referido Pacto. Diário Oficial da União, 22 fev. 2006a.

. Ministério da Saúde. Portaria no 648. Aprova a Política Nacional de Atenção Básica, estabelecendo a revisão de diretrizes e normas para a organização da Atenção Básica para o Programa Saúde da Família (PSF) e o Programa Agentes Comunitários de Saúde (Pacs). Diário Oficial da União, 28 mar. 2006b.

. Ministério da Saúde. Portaria no 649 . Define valores de financiamento para o ano de 2006, com vistas à estruturação de Unidades Básicas de Saúde para as equipes Saúde da Família, como parte da Política Nacional de Atenção Básica. Diário Oficial da União, 28 mar. 2006c.

. Ministério da Saúde. Portaria oㅡ 650. Define valores de financiamento do PAB fixo e variável mediante a revisão de diretrizes e normas para a organização da Atenção Básica, para a estratégia de Saúde da Família e para o Programa de Agentes Comunitários de Saúde, instituídos pela Política Nacional de Atenção Básica. Diário Oficial da União, 28 mar. 2006d. 
. Ministério da Saúde. Portaria no ${ }^{-698}$. Define que o custeio das ações de saúde é de responsabilidade das três esferas de gestão do SUS, observado o disposto na Constituição Federal e na Lei Orgânica do SUS. Diário Oficial da União, 30 mar. 2006e.

. Ministério da Saúde. Portaria nำ699. Regulamenta as Diretrizes Operacionais dos Pactos pela Vida e de Gestão. Diário Oficial da União, 30 mar. $2006 \mathrm{f}$.

CARVALHO, G. A inconstitucional administração pós-constitucional do SUS através de normas operacionais. Cienc. Saúde Coletiva, v. 6, n. 2, p. 435-444, 2001.

HORTALE, V. A.; PEDROZA, M.; ROSA, M. L. G. Operacionalizando as categorias acesso e descentralização na análise de sistemas de saúde. Cad. Saúde Pública, v. 16, n. 1, p. 231-239, 2000.

LAROUSSE. Dicionário Larousse ilustrado da língua portuguesa. São Paulo: Larousse do Brasil, 2004.

MANSUR, M. C. O financiamento federal da saúde no Brasil: tendências da década de 1990. 2001. Dissertação (Mestrado) — Escola Nacional de Saúde Pública, Fundação Oswaldo Cruz, Rio de Janeiro, 2001.

MARQUES, R. M.; MENDES, A. A política de incentivos do Ministério da Saúde para a atenção básica: uma ameaça à autonomia dos gestores municipais e ao princípio da integralidade? Cad. Saúde Pública, n. 18, p. 163-171, 2002. Suplemento.

MENDES, E. G. A descentralização do sistema de serviços de saúde no Brasil: novos rumos e um outro olhar sobre o nível local. In: MENDES, E. G. (Org.). A organização da saúde no nível local. São Paulo: Hucitec, 1998. p. 17-56.

ROMANO, C. S. O processo de municipalização da saúde no Brasil: considerações sobre uma política gradualista. In: CAMPOS, F. E.; OLIVEIRA, J. R.; TONON, L. M. (Orgs.). Cadernos de Saúde. Belo Horizonte: Coopmed, 1998. p. 1-9.

RONCALLI, A. G. O desenvolvimento das políticas públicas de saúde no Brasil e a construção do Sistema Único de Saúde. In: PEREIRA, A. C. et al. (Orgs.). Odontologia em saúde coletiva: planejando ações e promovendo saúde. Porto Alegre: Artmed, 2003. p. 28-49.

VIANA, A. L. A. et al. Mudanças significativas no processo de descentralização do sistema de saúde no Brasil. Cad. Saúde Pública, n. 18, p. 139-151, 2002. Suplemento. 